This item was submitted to Loughborough's Research Repository by the author.

Items in Figshare are protected by copyright, with all rights reserved, unless otherwise indicated.

\title{
The thermodynamic limit of the Whitham equations
}

PLEASE CITE THE PUBLISHED VERSION

https://doi.org/10.1016/S0375-9601(03)00515-2

PUBLISHER

(c) Elsevier

VERSION

AM (Accepted Manuscript)

PUBLISHER STATEMENT

This work is made available according to the conditions of the Creative Commons Attribution-NonCommercialNoDerivatives 4.0 International (CC BY-NC-ND 4.0) licence. Full details of this licence are available at: https://creativecommons.org/licenses/by-nc-nd/4.0/

\section{LICENCE}

CC BY-NC-ND 4.0

\section{REPOSITORY RECORD}

El, G.A.. 2019. "The Thermodynamic Limit of the Whitham Equations". figshare. https://hdl.handle.net/2134/32686. 


\title{
The thermodynamic limit of the Whitham equations
}

\author{
G.A. El \\ School of Mathematical and Information Sciences, Coventry University, Coventry, UK
}

\begin{abstract}
The infinite-genus limit of the KdV-Whitham equations is derived. The limit involves special scaling for the associated spectral surface such that the integrated density of states remains finite as $N \rightarrow \infty$ (the thermodynamic type limit). The limiting integro-differential system describes slow evolution of the density of states and can be regarded as the kinetic equation for soliton gas.
\end{abstract}

Keywords: modulation equations, finite-gap potentials, rotation number, thermodynamic limit

\section{Introduction}

As is well known [1, 2], the modulation (Whitham) equations for integrable systems describe gradual variations of spectral Riemann surfaces, whose local structure define $x, t$ - oscillating finite-gap potentials. The wave dynamics problems leading to the modulation equations are typically connected with the study of the long-time evolution of large-scale initial data [3], 4] and involve the semiclassical asymptotics of the inverse scattering transform. In this asymptotics, the typical scale of modulations $1 / \epsilon$ is necessarily proportional to a global number of degrees of freedom in the problem $g \gg 1$ (we assume no small parameters in the original equation, instead, we consider large-scale $\sim 1 / \epsilon$ initial data that can be approximated by $g$ soliton or $g$-gap potential). Then, asymptotically, the solution manifests itself as a modulated $N$-gap potential, where $N \ll g[5]$, [6]. The whole point of the traditional modulation theory, thus, is to reduce a complicated system with many degrees of freedom to a simpler one with a few degrees of freedom.

The modulation equations, however, can be considered on their own outside any relation to the semiclassical limit in the initial value problem. Then, at least formally, the question of possibility of the infinite-genus limit for the modulation equations can be risen. In this paper, I show that this limit, along with nontrivial mathematical meaning, has natural physical interpretation.

The paper is dedicated to the study of the limit as $N \rightarrow \infty$ for the $N$-gap averaged Korteweg - de Vries (KdV) equation. The $\mathrm{KdV}$ equation is taken in the form

$$
u_{t}-6 u u_{x}+u_{x x x}=0 .
$$

The key in the limiting transition for the modulation equations is the scaling of the spectral surface introduced by Venakides in the study of the continuum limit of theta-functions [7. The scaling implies that the essential part of the spectrum of the Shrödinger operator associated with (11) lies in a finite interval, say, $(-1,0)$ and the genus of the problem $N \gg 1$. 
Now, with the width of gaps being $O(1 / N)$ and the bands being exponentially narrow, $O(\exp (-N))$, the limit as $N \rightarrow \infty$ for the KdV-Whitham equations is shown to exist (Section $5)$. The existence of the limit for the Whitham equations is ultimately connected with the existence of the limit for the quantity called a 'rotation number' (or 'integrated density of states') introduced for almost periodic potentials by Johnson and Moser in 8. Since the established limit preserves finiteness of the density of states as $N \rightarrow \infty$, it can be called the thermodynamic limit. The limit of this type has been considered recently in [9] in connection with stochastic description of infinite-gap potentials.

The main result of the current paper is presented in Section 6 where the thermodynamic limit for the $\mathrm{KdV}$-Whitham equations is derived in the form of a nonlinear transport equation (51) for the density of states. The connection between 'density' and 'velocity' in (51) is given by the integral equation (52). I suggest that the obtained limit describes macroscopic dynamics of the 'thermodynamic limit of stochastic soliton lattices' considered in [9] and can be regarded as the kinetic equation for solitons. This interpretation is also supported by the exact agreement of the small density asymptotics of the resulting system with Zakharov's kinetic equation for a rarefied soliton gas [10].

\section{The Whitham equations: basic definitions}

In this section, we give a brief account on some basic definitions and notations from the finite-gap theory that will be used in this paper. The $\mathrm{KdV}$-Whitham system of genus $N$ can be represented as one generating equation in the Flashka-Forest-McLaughlin form [2]

$$
\begin{gathered}
\partial_{T} d p_{N}=\partial_{X} d q_{N}, \\
X=\epsilon x, \quad T=\epsilon t, \quad \epsilon \ll 1,
\end{gathered}
$$

where $d p_{N}$ and $d q_{N}$ are the meromorphic differentials on the hyperelliptic Riemann surface of genus $N$

$$
\begin{array}{ll}
\Gamma: \quad R^{2}(E)=\prod_{j=1}^{2 N+1}\left(E-E_{j}\right), & E \in \mathbf{C} \\
E_{1}<E_{2}<\ldots<E_{2 N}<E_{2 N+1}, & E_{j} \in \mathbf{R}
\end{array}
$$

with cuts along bands $\left[E_{2 j-1}, E_{2 j}\right]$. The canonical basis of cycles is organized as follows: the $\alpha_{j}$-cycle surrounds the $j$-th cut clockwise on the upper sheet, and the $\beta_{j}$ - cycle is canonically conjugated to $\alpha_{j}$ 's such that the closed contour $\beta_{j}$ starts at $E_{2 j}$, goes to $+\infty$ on the upper sheet and returns to $E_{2 j}$ on the lower sheet .

Then the meromorphic differentials are defiened by:

$$
\begin{gathered}
d p_{N}(E)=\frac{E^{N}+b_{N-1} E^{N-1}+\ldots+b_{0}}{R(E)} d E, \quad \oint_{\beta_{j}} d p_{N}(E)=0, \quad j=1, \ldots, N \\
d q_{N}(E)=12 \frac{E^{N+1}+c_{N} E^{N}+\ldots+c_{0}}{R(E)} d E, \quad c_{N}=-\frac{1}{2} \sum_{j=1}^{2 N+1} E_{j}
\end{gathered}
$$




$$
\oint_{\beta_{j}} d q_{N}(E)=0, \quad j=1, \ldots, N .
$$

The differentials $d p_{N}$ and $d q_{N}$ are often referred to as quasimomentum and quasienergy differentials. The integrals of $d p_{N}$ and $d q_{N}$ over the $\alpha$ - cycles are known to give the components of the wave number and the frequency vectors [2, [11]

$$
\oint_{\alpha_{j}} d p_{N}(E)=k_{j}, \quad \oint_{\alpha_{j}} d q_{N}(E)=\omega_{j}, \quad j=1, \ldots, N
$$

The finite-gap potentials associated with the Riemann surface (3) are known to be the multiphase solutions of the $\mathrm{KdV}$ equation (11) [12, [13, [2]

$$
u_{N}(x, t ; X, T)=u_{N}\left(y_{1}, \ldots, y_{N}\right)
$$

where the real phases

$$
y_{j}=k_{j} x+\omega_{j} t+f_{j}, \quad j=1, \ldots, N .
$$

are the linear function of 'fast' $x$ and $t$ while the dependence on 'slow' variables $X$ and $T$, enters the potential $u_{N}$ via the branch points $E_{j}(X, T)$ of the Riemann surface. The constant $f_{j}(\bmod 2 \pi)$ is the fixed initial phase. The function $u_{N}(\mathbf{y})$ is $2 \pi$-periodic in each phase $y_{j}$

$$
u_{N}\left(y_{1}, \ldots, y_{j}+2 \pi, \ldots, y_{N}\right)=u_{N}\left(y_{1}, \ldots, y_{j}, \ldots, y_{N}\right)
$$

that is $u_{N}(x, t)$ for fixed $E_{1}, \ldots E_{2 N+1}$ is $N$-quasiperiodic both in $x$ and $t$ (hereafter we consider only incommensurable $k_{j}$ s and $\omega_{j} \mathrm{~s}$ ).

The Whitham equations (2) thus describe slow evolution (modulations) of rapidly oscillating finite-gap potentials.

The algebro-geometric structure of the finite-gap potentials is given by the basis holomorphic differentials

$$
\psi_{j}=\sum_{k=0}^{N-1} a_{j k} \frac{E^{k}}{R(E)} d E, \quad j=1, \ldots, N .
$$

and the functions $a_{j k}\left(E_{1}, \ldots, E_{2 N+1}\right)$ are determined by the normalization over the $\alpha$-cycles

$$
\oint_{\alpha_{k}} \psi_{j}=\delta_{j k}
$$

while the integrals over the $\beta$-cycles give the entries of the Riemann matrix $B_{i j}$

$$
B_{i j}=\oint_{\beta_{j}} \psi_{i}
$$

The wavenumbers $k_{j}$ and the frequences $\omega_{j}$ are expressed in terms of $a_{i j}$ by the formulas [14, 2]

$$
\begin{gathered}
\mathbf{k}=-4 \pi i B^{-1} \mathbf{a}_{N-1} \\
\omega=\left(\omega_{1}, \ldots, \omega_{N}\right)=-8 \pi i B^{-1}\left(\mathbf{a}_{N-1} \sum_{j=1}^{2 N+1} E_{j}+2 \mathbf{a}_{N-2}\right) .
\end{gathered}
$$




\section{Rotation numbers and the density of states}

We consider the Schrödinger equation

$$
\left(-\partial_{x x}^{2}+q(x)\right) \psi=E \psi, \quad x \in \mathbf{R} .
$$

with an almost periodic potential $q(x)$. The potential $q(x)$ has an important characteristics, the rotation number, which was defined by Johnson and Moser [8] as

$$
\alpha(E)=\lim _{x \rightarrow \infty} \frac{1}{x} \arg \left(\psi^{\prime}(x, E)+i \psi(x, E)\right), \quad E \in \mathbf{R}
$$

As shown in [8], this agrees with the value $\pi \mathcal{N}$ where $\mathcal{N}(E)$ is the integrated density of states defined by

$$
\mathcal{N}(E)=\lim _{|b-a| \rightarrow \infty} \frac{\nu(a, b ; E)}{b-a},
$$

where $\nu(a, b ; E)$ is the number of eigenvalues $E_{j} \leq E$ in the Dirichlet problem on $a \leq x \leq b$ : $\psi(a, E)=\psi(b, E)=0$. Thus,

$$
\mathcal{N}(E)=\frac{1}{\pi} \alpha(E) .
$$

If, as a particular case, $q(x)$ is $N$-gap potential , $q(x)=u_{N}(x)$, such that $-1 \leq u_{N} \leq 0$ then the rotation number coincides with the real part of quasimomentum [8]:

$$
\alpha_{N}(E)=R e \int_{-1}^{E} d p_{N}\left(E^{\prime}\right), \quad E \in(-1,0),
$$

Then, with the aid of (6) we have

$$
\alpha_{N}(E)=\begin{array}{ll}
\frac{1}{2} \sum_{j=1}^{M(E)} k_{j} & \text { if } E \in \operatorname{gap}_{M} \\
\frac{1}{2} \sum_{j=1}^{M(E)} k_{j}+\int_{E_{2 M-1}}^{E} d p_{N}\left(E^{\prime}\right) \quad \text { if } E \in \operatorname{band}_{M},
\end{array}
$$

where $M(E)$ is the number of the band nearest to $E$ from the left; $M(E) \leq N, M(0)=$ $N, M(-1)=0$.

We note that the formula (19) agrees with the general statement in [8] that the values of the function $2 \alpha(E)$ if $E \in\{$ gap belong to the frequency-module of the almost periodic potential. One can also see that the function $\alpha^{\prime}(E)$ is positive and is supported on the spectrum of the finite-gap potential.

Since the finite-gap potential (7), (8) is an almost periodic function in $t$ as well as in $x$ one can also formally introduce a temporal analog of the rotation number

$$
\beta_{N}(E)=R e \int_{-1}^{E} d q_{N}\left(E^{\prime}\right)
$$


where $d q_{N}(E)$ is the quasienergy differential (5).

We also introduce the full integrated density of states $\kappa$ which is a number:

$$
\kappa=\mathcal{N}_{N}(0)=\frac{1}{2 \pi} \sum_{j=1}^{N} k_{j}
$$

One can see that the full integrated density of states has for the finite-gap potentials the natural significance of the mean number of waves per unit length.

Analogously, we introduce

$$
\Omega=\frac{1}{\pi} \beta_{N}(0)=\frac{1}{2 \pi} \sum_{j=1}^{N} \omega_{j}
$$

\section{Continuum limit of the spectrum and its thermody- namic nature}

Let the nontrivial (finite band) part of the spectrum lies in the interval $(-1,0)$ of the real $E$ axis: $\alpha_{N}(-1)=0, \alpha_{N}(0)=\pi \kappa$. For $N \gg 1$ we consider the special band/gap distribution (scaling):

$$
\operatorname{gaps}(E) \sim \frac{1}{N} \quad \operatorname{bands}(E) \sim \exp (-N) \quad N \gg 1, \quad E \in(-1,0) .
$$

Following Venakides [7] we introduce the lattice of points

$$
1 \approx \eta_{1}>\eta_{2}>\ldots>\eta_{N} \approx 0
$$

where

$$
-\eta_{j}^{2}=\frac{1}{2}\left(E_{2 j-1}+E_{2 j}\right)
$$

are centers of bands.

We define two positive continuous functions on $[0,1]$ :

1. The normalized density of bands $\varphi(\eta)$ :

$$
\varphi(\eta) d \eta \approx \frac{\text { number of lattice points in }(\eta, \eta+d \eta)}{N}
$$

That is,

$$
\varphi\left(\eta_{j}\right)=\frac{1}{N\left(\eta_{j}-\eta_{j+1}\right)}+O\left(\frac{1}{N}\right), \quad \int_{0}^{1} \varphi(\eta) d \eta=1, \quad \eta^{2}=-E \in(0,1) .
$$

2. The normalized logarithmic band width $\gamma(\eta)$ :

$$
\gamma\left(\eta_{j}\right)=-\frac{1}{N} \log \delta_{j}+O\left(\frac{1}{N}\right), \quad \delta_{j}=E_{2 j}-E_{2 j-1} .
$$

The functions $\varphi(\eta)$ and $\gamma(\eta)$ asymptotically define the local structure of the Riemann surface $\Gamma$ (3) for $N \gg 1$. In other words, instead of $2 N+1$ discrete parameters $E_{j}$ we have two 
continuous functions of $\eta$ on $(-1,0)$, which do not depend on $x, t$ on $\Delta x \sim \Delta t \sim 1$. On a larger scale, $\Delta x \sim \Delta t \sim 1 / \epsilon, \epsilon \ll 1$, in the spirit of the modulation theory, we put

$$
\varphi=\varphi(\eta ; X, T) \quad \gamma=\gamma(\eta ; X, T),
$$

where $X=\epsilon x, T=\epsilon t$. These functions are now subject to the evolution equations which naturally should be derived as the continuum limit of the Whitham equations (2) considered for the same exponential spectral scaling (23).

In the next section we will show that the scaling (23) implies the following asymptotic behaviour for the wavenumbers and the frequences (these asymptotics can also be derived directly from (6) ):

$$
\begin{aligned}
k_{j} \sim \omega_{j} & \sim\left|\frac{1}{\log \delta_{j}}\right|=O\left(\frac{1}{N}\right), \quad N \gg 1, \\
\int_{E_{2 j-1}}^{E} d p_{N}\left(E^{\prime}\right) & \sim \int_{E_{2 j-1}}^{E} d q_{N}\left(E^{\prime}\right)<O\left(\frac{1}{N}\right), \quad E \in\left(E_{2 j-1}, E_{2 j}\right),
\end{aligned}
$$

which provides finiteness of the rotation number $\alpha_{N}(E)$ (and of the integrated density of states $\left.\mathcal{N}_{N}(E)\right)$ as $N \rightarrow \infty$ :

$$
\alpha(E) \equiv \lim _{N \rightarrow \infty} \alpha_{N}(E)=\lim _{N \rightarrow \infty} \frac{1}{2} \sum_{j=1}^{M(E)} k_{j}<\infty, \quad M \leq N
$$

Due to this property we call the scaling (23) and the corresponding limit as $N \rightarrow \infty$ the thermodynamic ones. This type of limits in the finite-gap theory has been introduced in [9]. Hereafter we will denote the limit of some functional $f\left[u_{N}\right]$ as $N \rightarrow \infty$ on the thermodynamic spectral scaling as

$$
T-\lim f\left[u_{N}\right]
$$

It should be noted that the thermodynamic spectral scaling (23) appears when one considers the semiclassical asymptotics of the spectrum for periodic potentials [15, 4], [7]. However, in the works just cited, $N \sim 1 / \epsilon$, which is the standard relation in the asymptotics of this type (see also 3]). Contrastingly, in the thermodynamic limiting transition, the large parameter $N$ defining the scaling is contained only in the band-gap structure and is not connected with the parameter $1 / \epsilon$, which scales modulations of the finite-gap potential $u_{N}(x, t)$.

\section{The thermodynamic limit for the rotation numbers: basic integral equations}

Our aim now is to deduce the thermodynamic limit for the modulation equation (2). For that, we need to evaluate the thermodynamic limits of the meromorphic differentials $d p_{N}$ and $d q_{N}$. First we make an estimate for their imaginary parts on the thermodynamic scaling (23): 


$$
\begin{aligned}
& 0 \quad E \in \text { any band } \\
& \left|\operatorname{Im} \int_{-1}^{E} d p_{N}\left(E^{\prime}\right)\right|= \\
& \left|\int_{E_{2 j}}^{E} d p_{N}\left(E^{\prime}\right)\right|<O\left(\frac{1}{N}\right) \quad E \in \operatorname{gap}_{j} .
\end{aligned}
$$

Therefore, the imaginary part of $d p_{N}(E)$ vanishes as $N \rightarrow \infty$ and we have for $E \in(-1,0)$ :

$$
T-\lim p_{N}(E)=\alpha(E)=T-\lim \frac{1}{2} \sum_{j=1}^{M(E)} k_{j} .
$$

Analogously, we get

$$
T-\lim q_{N}(E)=\beta(E)=T-\lim \frac{1}{2} \sum_{j=1}^{M(E)} \omega_{j},
$$

where $\beta(E)=T-\lim \beta_{N}(E)$.

Now we evaluate the wave numbers $k_{j}$ and the frequencies $\omega_{j}$ for the chosen spectral scaling. For that, we will make use of the following Venakides observation [7: for the spectral distribution (23) the following approximation for the basis holomorphic differentials is valid:

$$
\psi_{j} \approx-\frac{\eta_{j}}{2 \pi} \frac{\prod_{i=1, i \neq j}^{N}\left(E+\eta_{j}^{2}\right)}{\left(\prod_{k=1}^{2 N+1}\left(E-E_{j}\right)\right)^{1 / 2}} d E .
$$

One can see that

$$
\left(E-E_{2 j-1}\right)\left(E-E_{2 j}\right)=\left(E+\eta_{j}^{2}\right)+O\left(e^{-N}\right) .
$$

Then an extensive cancellation becomes possible in (34) so that the hyperelliptic integrals in (11), (12) turn into elementary ones. As a result, we have the following asymptotics (see [7] for details):

$$
\begin{gathered}
B_{i j} \approx-\frac{i}{\pi}\left(\log \left|\frac{\eta_{i}-\eta_{j}}{\eta_{i}+\eta_{j}}\right|+N \gamma\left(\eta_{i}\right) \delta_{i j}\right), \quad i, j=1, \ldots, N . \\
a_{j, N-1} \approx-\frac{\eta_{j}}{2 \pi}, \quad a_{j, N-1} \sum_{j=1}^{2 N+1} E_{j}+2 a_{j, N-2} \approx \frac{\eta_{j}^{3}}{\pi} .
\end{gathered}
$$

Now we represent the general relationships (13) between the real and imaginary periods of theta function in the form convenient for the thermodynamic limiting transition

$$
\begin{gathered}
B \mathbf{k}=-4 \pi i \mathbf{a}_{N-1} \\
B \omega=-8 \pi i\left(\mathbf{a}_{N-1} \sum_{j=1}^{2 N+1} E_{j}+2 \mathbf{a}_{N-2}\right) .
\end{gathered}
$$


It follows from the asymptotics (35), (36) that to provide a balance of terms in (37), (38) the following scaling is necessary : $k_{j} \sim \omega_{j} \sim O(1 / N)$. Therefore, we introduce two continuous on $(0,1)$ functions $k(\eta)$ and $\omega(\eta)$ by

$$
k_{j}=\frac{1}{N} k\left(\eta_{j}\right), \quad \omega_{j}=\frac{1}{N} \omega\left(\eta_{j}\right) .
$$

Then, substituting (35), (36), (39), into (37), (38) we get two algebraic systems for $k\left(\eta_{i}\right)$, $\omega\left(\eta_{i}\right), i=1, \ldots, N$

$$
\begin{gathered}
\sum_{j=1}^{N} \frac{1}{N} \log \left|\frac{\eta_{i}-\eta_{j}}{\eta_{i}+\eta_{j}}\right| k\left(\eta_{j}\right)+\gamma\left(\eta_{i}\right) k\left(\eta_{i}\right)=-2 \pi \eta_{i} . \\
\sum_{j=1}^{N} \frac{1}{N} \log \left|\frac{\eta_{i}-\eta_{j}}{\eta_{i}+\eta_{j}}\right| \omega\left(\eta_{j}\right)+\gamma\left(\eta_{i}\right) \omega\left(\eta_{i}\right)=8 \eta_{i}^{3} .
\end{gathered}
$$

Using (39) we represent the relationships (32), (33) for the thermodynamic limit of the rotation numbers in a continuum form:

$$
2 d \alpha\left(-\eta^{2}\right)=\varphi(\eta) k(\eta) d \eta, \quad 2 d \beta\left(-\eta^{2}\right)=\varphi(\eta) \omega(\eta) d \eta,
$$

Now one readily obtains the thermodynamic limit of (401), (411) :

$$
\begin{gathered}
\int_{0}^{1} \log \left|\frac{\eta-\mu}{\eta+\mu}\right| \frac{\mu}{\eta} \alpha^{\prime}\left(-\mu^{2}\right) d \mu+\sigma(\eta) \alpha^{\prime}\left(-\eta^{2}\right)=\frac{\pi}{2} . \\
\int_{0}^{1} \log \left|\frac{\eta-\mu}{\eta+\mu}\right| \frac{\mu}{\eta} \beta^{\prime}\left(-\mu^{2}\right) d \mu+\sigma(\eta) \beta^{\prime}\left(-\eta^{2}\right)=-2 \pi \eta^{2},
\end{gathered}
$$

where

$$
\sigma(\eta)=\frac{\gamma(\eta)}{\varphi(\eta)} .
$$

We see that the thermodynamic limit of the rotation numbers is determined by the only function $\sigma(\eta)$ defined on $(0,1)$ (instead of the original two: $\varphi(\eta)$ and $\gamma(\eta)$ ).

The integral equations (43), (44) have been derived in [9] in connection with the establishing the thermodynamic limit for the stochastic processes generated by the finite-gap potentials (stochastic soliton lattices). We note that these integral equations also appear in [16] where they determine the Lax-Levermore type minimizer for the $N$-soliton solution with randomly distributed soliton phases as $N \rightarrow \infty$. It is clear that there should be a direct connection between the 'stochastic' version of the Lax-Levermore variational problem and the thermodynamic limit of the rotation number.

\section{Thermodynamic limit of the Whitham equations}

The modulation equation (2) describe slow $X, T$-deformations of the spectral surface (3). Now we are going to apply the thermodynamic limiting transition to (2). As follows from (32), (33), the local thermodynamic limits are

$$
T-\lim d p_{N}(E)=d \alpha(E) \quad T-\lim d q_{N}(E)=d \beta(E) .
$$


We put that on a larger scale

$$
d \alpha=d \alpha\left(-\eta^{2} ; X, T\right) \quad d \beta=d \beta\left(-\eta^{2} ; X, T\right) .
$$

Then the thermodynamic limit of the Whitham system (21) takes the form:

$$
\partial_{T} \alpha^{\prime}=\partial_{X} \beta^{\prime}
$$

where the functional dependence $\beta^{\prime}\left[\alpha^{\prime}\left(-\eta^{2} ; X, T\right)\right]$ is given by (43), (44). The thermodynamic limit of the Whitham equations, therefore, describes evolution of the density of states $\alpha^{\prime}(E) / \pi$.

Since $d \alpha(E)>0$ one can introduce a distribution function $f(\eta ; X, T)$ by

$$
f(\eta ; X, T) d \eta=\frac{1}{\pi} d \alpha\left(-\eta^{2} ; X, T\right), \quad \int_{0}^{1} f d \eta=\kappa,
$$

Introducing

$$
s(\eta ; X, T)=\frac{\beta^{\prime}\left(-\eta^{2} ; X, T\right)}{\alpha^{\prime}\left(-\eta^{2} ; X, T\right)}
$$

one can represent (48) in the form of a nonlinear transport equation:

$$
\partial_{T} f=\partial_{X}(s f)
$$

where the integral equation connecting $s$ with $f$ is obtained by excluding $\sigma(\eta)$ from (43), (44):

$$
s(\eta)=-4 \eta^{2}+\frac{1}{\eta} \int_{0}^{1} \log \left|\frac{\eta-\mu}{\eta+\mu}\right| f(\mu)[s(\mu)-s(\eta)] d \mu .
$$

Here, of course, $s(\eta) \equiv s(\eta ; X, T), f(\eta) \equiv f(\eta ; X, T)$. In the case of small integrated density of states $\kappa \ll 1$ we get

$$
s \approx-4 \eta^{2}-\frac{4}{\eta} \int_{0}^{1} \log \left|\frac{\eta-\mu}{\eta+\mu}\right| f(\mu)\left[\mu^{2}-\eta^{2}\right] d \mu,
$$

which is Zakharov's expression for the velocity of the 'trial' soliton moving through rarefied soliton gas [10]. The integro-differential system (51), (52), therefore, can be regarded as the finite-density generalisation of the Zakharov kinetic equation for solitons.

Now we discuss briefly what happens to the underlying finite-gap oscillating microstructure under the thermodynamic limiting transition. It has been shown in [9] that the thermodynamic limit of the finite-gap potentials should be treated as a random process. More accurately, the thermodynamic limit is defined not for the individual potential $u_{N}(x)$ but for the stationary random process generated by considering the associated torus equipped with the Haar measure. Such processes were called in [17] stochastic soliton lattices. The stochastic soliton lattice $\nu(x \mid \phi)$ is defined by

$$
\nu_{N}(x \mid \phi)=u_{N}\left(\ldots, k_{j} x+\phi_{j}, \ldots\right), \quad j=1, \ldots, N
$$


where $\phi=\left(\phi_{1}, \ldots, \phi_{N}\right)$ is a random value uniformly distributed on $\operatorname{Tor}^{N}$. It was shown in [9] that the thermodynamic limit of the stochastic soliton lattice

$$
T-\lim \nu_{N}(x \mid \phi)=\zeta\left(x-x_{j}\right)
$$

represents a random process defined on the infinite discrete set of points $\left\{x_{j} ; j \in \mathbf{Z}\right\}$ distributed by Poisson on $\mathbf{R}$. Since the band/gap ratio for the chosen spectral scaling is proportional to $N e^{-N}$, it vanishes as $N \rightarrow \infty$ and the thermodynamic limit of the stochastic soliton lattices (55) can be associated with one-dimensional soliton gas. This also supports our interpretation of the thermodynamic limit of the Whitham equations as the kinetic equation for solitons.

\section{$7 \quad$ Averaged conservation laws}

Integrating (48) by $E$ over the interval $(-1,0)$ we obtain the thermodynamic limit of the wave conservation law:

$$
\partial_{T} \kappa=\partial_{X} \Omega
$$

where $\pi \kappa$ is the full density of waves (integrated wavenumber) (21) and $\pi \Omega$ is the corresponding 'integrated frequency'(see (22) ).

The quasimomentum $p_{N}(E)$ is known to be the generating function for the Kruskal integrals $I_{k}^{(N)}$ [1], [8]:

$$
\begin{gathered}
p_{N}(E)=2 \sqrt{-E}\left(1+\sum_{k=0}^{\infty} \frac{I_{k}^{(N)}}{(-2 E)^{k}}\right), \quad-E \gg 1, \\
I_{0}^{(N)}=\lim _{L \rightarrow \infty} \frac{1}{L} \int_{0}^{L} u_{N}(x) d x, \quad I_{1}^{(N)}=\lim _{L \rightarrow \infty} \frac{1}{L} \int_{0}^{L} \frac{1}{2} u_{N}^{2}(x) d x, \ldots
\end{gathered}
$$

Then the thermodynamic limit for the Kruskal integrals is calculated as

$$
\begin{gathered}
P_{k}(X, T)=T-\lim I_{k}^{(N)}=T-\lim \left(\operatorname{Res}_{-\infty}\left[\frac{2^{2 k+1}}{2 k+1} \frac{d p_{N}}{d E}(-E)^{\frac{2 k+1}{2}}\right]\right) \\
=\frac{2^{2 k+1}}{(2 k+1) \pi}(-1)^{k+1} \int_{-1}^{0}(-E)^{\frac{2 k+1}{2}} \alpha^{\prime}(E ; X, T) d E \\
=\frac{2^{2 k+1}}{2 k+1}(-1)^{k+1} \int_{0}^{1} \eta^{2 k+1} f(\eta ; X, T) d \eta, \quad k=0,1,2, \ldots
\end{gathered}
$$

Thus, the infinite set of the independent averaged conservation laws takes the form

$$
\partial_{T} P_{k}=\partial_{X} Q_{k}, \quad k=0,1,2 \ldots,
$$

where the 'fluxes' $Q_{k}(X, T)$ are found analogously to (59) from the thermodynamic limit for the quasienergy differential (46):

$$
Q_{k}(X, T)=\frac{2^{2 k+1}}{2 k+1}(-1)^{k+1} \int_{0}^{1} \eta^{2 k+1} f(\eta ; X, T) s(\eta ; X, T) d \eta .
$$


In particular, it follows from (158), (59) that the thermodynamic limits for the first two KdV moments are

$$
\bar{u}(X, T)=-2 \int_{0}^{1} \eta f(\eta ; X, T) d \eta, \quad \overline{u^{2}}(X, T)=\frac{16}{3} \int_{0}^{1} \eta^{3} f(\eta ; X, T) d \eta .
$$

We note in conclusion, that it is clear that the procedure of the thermodynamic limiting transition can be extended to other integrable Whitham hierarchies. Another implication of the developed theory is the thermodynamic limit for the Krichever algebro-geometrical

procedure of integration of the Whitham equations [18, [11]. This result will be published in a separate paper [19.

\section{Aknowledgements}

I would like to thank Alexander Krylov for his permanent interest in this work and numerous useful discussions. I am also grateful to Martin Kruskal for his inspiring comments at the NEEDS 2002 meeting where part of the results of this work has been reported.

\section{References}

[1] G.B. Whitham, Proc.Roy.Soc. A283 (1965) 238.

[2] H. Flaschka, G. Forest, D.W. McLaughlin, Comm. Pure Appl. Math. 33 (1979) 739.

[3] P.D.Lax and C.D.Levermore, Comm. Pure Appl. Math. 36 (1983) 253,571,809.

[4] S.Venakides, T. Am.Math.Soc. 301(1987)189

[5] S.Venakides, Comm. Pure Appl. Math. 43 (1990) 335.

[6] P. Deift, S. Venakides, and X. Zhou, International Math. Research. Journ., no 4, (1997) 285.

[7] S. Venakides, Comm.Pure Appl.Math. 42 (1989) 711.

[8] R.Johnson and J.Moser, Comm.Math.Phys. 84 (1982) 403.

[9] G.A.El, A.L.Krylov, S.Molchanov, S.Venakides, Physica D 152-153 653

[10] V.E.Zakharov, Sov.Phys.JETP 60 (1971) 1012.

[11] B.A.Dubrovin and S.P.Novikov, Russian Math. Surveys 44 (1989) 35 .

[12] S.P. Novikov, Func. Anal.Pril. 8 (1974) 54.

[13] P.D. Lax, Comm.Pure Appl. Math. 26 (1975) 141.

[14] A.R.Its and V.B.Matveev, Theor.Math.Phys. 23 (1975) 343.

[15] M.I.Weinstein, J.B.Keller, SIAM Jornal Appl.Math. 47(1987)941. 
[16] A.V. Gurevich, N.G. Mazur, K.P. Zybin JETP 90 (2000) 695.

[17] A.L.Krylov, G.A. El, Soviet Math. Surveys 54 (1999) 439.

[18] I.M.Krichever, Func. Anal. Appl. 22 (1988) 200.

[19] G.A.El, Algebro-geometrical solutions of the infinite-genus Whitham eqations, (to be published). 